BMJ Open Sport \& Exercise Medicine

\title{
Sleep deprivation and obesity in adults: a brief narrative review
}

Christopher B Cooper, ${ }^{1}$ Eric V Neufeld, ${ }^{1}$ Brett A Dolezal, ${ }^{1}$ Jennifer L Martin ${ }^{2,3}$

To cite: Cooper CB, Neufeld EV, Dolezal BA, et al. Sleep deprivation and obesity in adults: a brief narrative review. BMJ Open Sport \& Exercise Medicine 2018;4:e000392. doi:10.1136/ bmjsem-2018-000392

Accepted 6 August 2018
Check for updates

(C) Author(s) (or their employer(s)) 2018. Re-use permitted under CC BY-NC. No commercial re-use. See rights and permissions. Published by BMJ.

${ }^{1}$ Exercise Physiology Research Laboratory, Departments of Medicine and Physiology, David Geffen School of Medicine at University of California, Los Angeles, California, USA ${ }^{2}$ Department of Medicine, David Geffen School of Medicine at University of California, Los Angeles, California, USA ${ }^{3} \mathrm{VA}$ Greater Los Angeles Healthcare System, Geriatric Research, Education and Clinical Center, Los Angeles, California, USA

Correspondence to Dr Christopher B Cooper; ccooper@mednet.ucla.edu

\section{ABSTRACT}

Background/aims Obesity and sleep deprivation are two epidemics that pervade developed nations. Their rates have been steadily rising worldwide, especially in the USA This short communication will explore the link between the two conditions and outline the proposed mechanisms behind their relationship.

Methods Studies on the topic of sleep and obesity were reviewed, and findings were used to develop a theoretical model for the biological link between short sleep duration and obesity.

Results Individuals who regularly slept less than 7 hours per night were more likely to have higher average body mass indexes and develop obesity than those who slept more. Studies showed that experimental sleep restriction was associated with increased levels of ghrelin, salt retention and inflammatory markers as well as decreased levels of leptin and insulin sensitivity.

Conclusions There may be a link between obesity and sleep deprivation. We recommend further investigations are to elucidate the potential mechanisms.

\section{INTRODUCTION}

Sleep deprivation occurs when an individual's biological sleep need is not met. In epidemiological studies, definitions vary, but sleep deprivation is typically considered obtaining less than 7 hours of sleep. There are numerous studies in the scientific literature that suggest that sleep deprivation has metabolic effects that predispose to weight gain. Currently, developed nations are facing an epidemic of obesity. For example, the prevalence of obesity in the USA increased from $22.9 \%$ in $1988-1994$ to $37.7 \%$ in $2013-2014 .{ }^{1}$ By 2014 , the rate of obesity had reached $35.0 \%$ among adult men and $40.4 \%$ among adult women. Also, data indicate that the adult population in the USA is getting less sleep, ${ }^{2}$ and a significant proportion receives less than the recommended 7 hours of sleep per night. ${ }^{3}$ In 1998, $26 \%$ of people reported sleeping less than 8 hours, whereas in 2005, the proportion was $35 \%$. In the National Health Interview survey of 110442 civilian employed workers between 2004 and 2007, the weighted prevalence of self-reported short sleep duration, defined as $\leq 6$ hours per day, was $29.9 \%{ }^{4}$

\section{What is already known?}

There is a bidirectional link between sleep deprivation and obesity.

\section{What are the new findings?}

Sleep deprivation may mediate increases in body mass index through elevated ghrelin, suppressed leptin and augmented hedonic signalling during food intake.

- Decreased sleep results in increased fatigue, which may lower capability for exercise.

- Obesity increases the risk for sleep disorders, which may compromise sleep quality.

\section{METHODS}

The electronic search strategy was created and completed by a single researcher (CBC), and the results were reviewed by the other members of the research team. A computerised literature search was performed using PubMed/MEDLINE and Google Scholar with keywords 'sleep deprivation', 'sleep restriction', 'short sleep', 'sleep duration', 'obesity', 'weight gain' and 'body mass index'. Titles and abstracts of potentially relevant articles published in English between 2000 and 2017 were screened. Studies were included solely based on relevance to the research question. Included papers were grouped based on content and methodology into one of the following categories: prospective studies (single investigations and reviews/ meta-analyses), cross-sectional studies (single investigations and reviews/meta-analyses), mixed studies (single investigations and reviews/meta-analyses) and mechanistic studies (single investigations and reviews/ meta-analyses). Prospective, cross-sectional and mixed studies examined the epidemiological relationship between sleep deprivation and obesity, while mechanistic studies offered insight on the mechanisms driving the relationship between the two. 


\section{RESULTS}

A systematic literature search for epidemiological evidence of an association between sleep deprivation and obesity provided 92 original studies and 18 reviews of various subsets of these investigations.

\section{Prospective studies}

Two meta-analyses, one of 30 pooled studies (634 511 participants) and the other of 11 studies (197 906 participants), demonstrated that short sleep is associated with an increase in body mass index (BMI) and risk of developing obesity. Similar findings, including in a cohort solely comprised of women, were reported up to a certain age. Associations between sleep deprivation and obesity diminished after 27 years of age in one investigation and disappeared in women 40 years and older in another. In contrast, a different study concluded that older adults who slept less than 5 hours compared with $7-8$ hours increased their risk of obesity by $40 \%$. Other analysis calculated an increased risk of $15 \%$ in individuals sleeping 5 hours or less and $6 \%$ in those sleeping 6 hours compared with those sleeping 7-8 hours.

\section{Cross-sectional studies}

One cross-sectional study of 41610 participants reported an association between weight change (defined as gaining or losing $\geq 5 \mathrm{~kg}$ ) within 5 years and an increased likelihood to report decreased total sleep time. Individuals more likely to report decreased total sleep time were women who lost significant weight as well as both men and women who gained significant weight.

\section{Mixed studies}

Mixed studies were those that included both prospective and cross-sectional analyses or a review of both prospective and cross-sectional studies. Several investigations revealed that individuals with short sleep duration had higher average BMI and were at greater risk for developing obesity. In addition to obesity, one meta-analysis of 12 pooled studies (18 720 patients with metabolic syndrome and 70833 healthy controls) found a link between sleep deprivation and an increased risk for developing metabolic syndrome. Other studies, however, reported mixed results in older adults and concluded that conflicting mathematical relationships between sleep deprivation and obesity suggest an insufficient body of evidence to establish a link between the two.

\section{Mechanistic studies}

Experimental sleep restriction has been associated with increased levels of ghrelin, salt retention and inflammatory markers as well as decreased levels of leptin and insulin sensitivity. Higher ghrelin and lower leptin following sleep deprivation were correlated with increased hunger, especially for foods dense in fats and carbohydrates. Furthermore, consumption of these calories was found to increase the activity of neuronal reward pathways. Other studies revealed decreased thyroid-stimulating hormone (TSH) and free thyroxine $\left(\mathrm{T}_{4}\right)$ following chronic partial sleep loss. Behavioural mechanisms, such as reduced physical activity secondary to increased fatigue, irregular feeding and increased feeding due to more time spent awake, were also suggested as possible links between sleep deprivation and obesity.

\section{DISCUSSION}

\section{Prospective studies}

In one meta-analysis of 30 investigations (634 511 participants), a pooled regression analysis in the adults suggested that a reduction in 1 hour of sleep per day would be associated with a $0.35 \mathrm{~kg} / \mathrm{m}^{2}$ increase in BMI. For a person approximately $178 \mathrm{~cm}$ tall that would be equivalent to a weight gain of approximately $1.4 \mathrm{~kg}(3.1$ lbs). The authors explained that insufficient evidence exists to draw conclusions about cause and effect from this literature. ${ }^{5}$ A prospective study was conducted in 596 young adults who were interviewed at ages 27, 29, 34 and. $^{6}$ An association was found between short sleep duration and obesity at age 27 years, but this association diminished with age. Similarly, a 10-year longitudinal investigation of 4903 women reported a significant link between short sleep duration and the risk for developing obesity in those younger than 40 years but not in women 40 years or older. ${ }^{7}$ A different epidemiological study of older adults concluded that sleep duration of less than 5 hours, compared with sleeping 7-8 hours, increased the likelihood of developing obesity by $40 \%{ }^{8}$ These results were echoed by a meta-analysis of 11 prospective studies (197 906 participants) that also found a significant association in both sexes between short sleep duration (defined as less than 5-6 hours per night) and the risk for developing obesity. ${ }^{9}$

The prospect of future weight gain with restricted sleep was investigated in the Nurses Health Study. ${ }^{10}$ The authors originally enrolled 121700 female nurses in 1976. The cohort was surveyed in 1986 and asked about sleep duration. Overall, $4.3 \%$ of women slept 5 hours or less, $25.5 \%$ slept 6 hours, $42.1 \%$ slept 7 hours, $23.5 \%$ slept 8 hours and $4.5 \%$ slept 9 hours or more. Respondents were then contacted every 2 years for up to 16 years and asked to report their body weight. Voluntary physical activity levels were similar between groups.

At baseline, there was a clear cross-sectional relationship between weight and sleep duration with those sleeping 5 hours or less weighing on average $2.47 \mathrm{~kg}$ ( $5.43 \mathrm{lbs}$ ) more than those sleeping 7 hours (the median for the cohort) and $1.24 \mathrm{~kg}$ (2.73 lbs) more than those sleeping 6 hours. Furthermore, while all groups gained weight over the ensuing 10 years, weight increased more rapidly in those sleeping the least. Those sleeping 5 hours or less gained $0.73 \mathrm{~kg}(1.61 \mathrm{lbs})$ more and those sleeping 6 hours gained $0.26 \mathrm{~kg}(0.57 \mathrm{lbs})$ more than those sleeping 7 hours. Over the 16 years, $10.5 \%$ of the women being followed gained $15 \mathrm{~kg}$ (33 lbs) or more.

Women sleeping 5 hours or less were $32 \%$ more likely and those sleeping 6 hours were $12 \%$ more likely to gain this amount of weight compared with those sleeping 7-8 
hours. Of those women who were not obese at baseline, $15.9 \%$ had become obese at the end of 16 years as defined by a BMI $\geq 30 \mathrm{~kg} / \mathrm{m}^{2}$. The risk of developing obesity was increased by $15 \%$ in those sleeping 5 hours or less and increased by $6 \%$ in those sleeping 6 hours compared with those sleeping 7-8 hours. The comparisons described above were statistically significant, and in the statistical analysis reported by these investigators, all models were adjusted for potential confounding variables such as age, smoking status, alcohol consumption, caffeine consumption and the use of medications known to affect sleep.

\section{Cross-sectional studies}

One cross-sectional study of 41610 participants found that both men and women who experienced significant weight gain (defined as 5 or more kilograms within 5 years) were more likely to report a decreased total sleep time than those of stable weight. Interestingly, women who lost significant weight were also more likely to report decreased total sleep time. This same trend was not observed in men. The authors noted, however, that the composition of the weight gained or lost was not specified. ${ }^{11}$

\section{Mixed studies}

One review reported that short sleep duration was consistently linked with the development of obesity in children and young adults; findings in older adults were mixed. ${ }^{12}$ A meta-analysis of 12 studies (18 720 patients with metabolic syndrome and 70833 healthy controls) found a significant association in both sexes between short sleep duration (defined as less than 5-6 hours per night) and an increased risk for developing metabolic syndrome. ${ }^{13}$ Examination of the database from the National Health and Nutritional Examination Survey I between 1982 and 1992 revealed that subjects between the ages of 32 years and 49 years with self-reported sleep durations at baseline less than 7 hours had higher average BMIs and were more likely to be obese than subjects with sleep durations of 7 hours. ${ }^{14}$ This analysis raised awareness of the possibility of a connection between sleep restriction and the development of obesity. On the contrary, a different review concluded that there is inadequate evidence to link decreased sleep duration with the increasing incidence of obesity. The authors reported that the reviewed cross-sectional studies yielded both inverse and zero relationships between sleep duration and obesity. Reviewed longitudinal studies yielded similar mixed results. ${ }^{15}$

\section{Mechanistic studies}

Experimental studies have shown sleep restriction to influence two important hormones, leptin and ghrelin, that regulate metabolism and energy expenditure. ${ }^{16}$ Leptin is released from adipose tissue (fat) and acts on receptors in the hypothalamus of the brain where it inhibits appetite and promotes satiety thus limiting food intake. Circulating blood levels of leptin are generally proportional to body fat mass. Ghrelin, however, is released from the stomach and pancreas and stimulates appetite. Circulating ghrelin levels fluctuate over the course of the day in relation to food intake. Leptin is decreased with sleep deprivation, ${ }^{17}$ whereas ghrelin is increased. ${ }^{18}$ In a short-term study of 10 men, 2 days of sleep restriction was associated with an $18 \%$ reduction in the leptin and a 28\% elevation in ghrelin. These changes were associated with increased hunger and appetite, especially for calorie-dense foods with high carbohydrate content. ${ }^{18}$ Both the decrease in leptin and the increase in ghrelin seen with sleep deprivation could potentially increase food intake and contribute to weight gain. ${ }^{19}$ Considering that increased caloric intake has been shown to promote sleep, sleep deprivation may prompt overeating as a compensatory mechanism to regain lost sleep. ${ }^{20}{ }^{21}$ This response may be governed on a neurological level as increased activity of neuronal reward pathways (hedonic signalling) during food intake has been observed following sleep deprivation. ${ }^{21}$ These and other potential mechanisms by which sleep deprivation may predispose to obesity are summarised in figure 1 .

In addition to biological associations, several authors have highlighted the potential for behavioural mechanisms between sleep deprivation and obesity. The most straightforward of these states that individuals who sleep less have more opportunities to consume calories. Short sleepers may also experience fatigue, which reduces the likelihood of engaging in physical activity. ${ }^{22}$ One review noted that decreased sleep allowed for the possibility of increased feeding late at night and early in the morning. ${ }^{23}$ Considering that components of metabolism can differ depending on the time of day, it is reasonable to believe that caloric intake during these periods may result in altered metabolic responses. ${ }^{23}$ Furthermore, women may respond differently to disruptions in sleep duration and metabolism than men, but little research exists on this topic. ${ }^{21}$

Cross-sectional and longitudinal epidemiological studies have also shown associations between short sleep duration and diabetes, ${ }^{24}{ }^{25}$ hypertension, ${ }^{26}{ }^{27}$ cardiovascular disease ${ }^{28} 29$ and mortality. ${ }^{6}$ Habitually short sleep duration could lead to insulin resistance by increasing sympathetic nervous system activity, raising evening cortisol levels and decreasing cerebral glucose utilisation that over time could compromise pancreatic beta-cell function and lead to diabetes. One review reported that both acute and chronic sleep restriction impaired insulin sensitivity by $20 \%-30 \%$, which lasted between 1 day and 2 weeks. ${ }^{30}$ Another stated that sleep deprivation reduced both glucose tolerance (insulin-mediated uptake) and glucose effectiveness (insulin-independent uptake). ${ }^{31}$ It has been postulated that sleep restriction may also indirectly affect insulin secretion through its modulatory effects on leptin and ghrelin. ${ }^{30} 32$ Other endocrine signalling affected includes the hypothalamic-pituitarythyroid axis. $\mathrm{TSH}^{3133}$ and free $\mathrm{T}_{4}^{33}$ levels were decreased following chronic partial sleep loss suggesting a possible corresponding reduction in metabolic rate. However, it is 


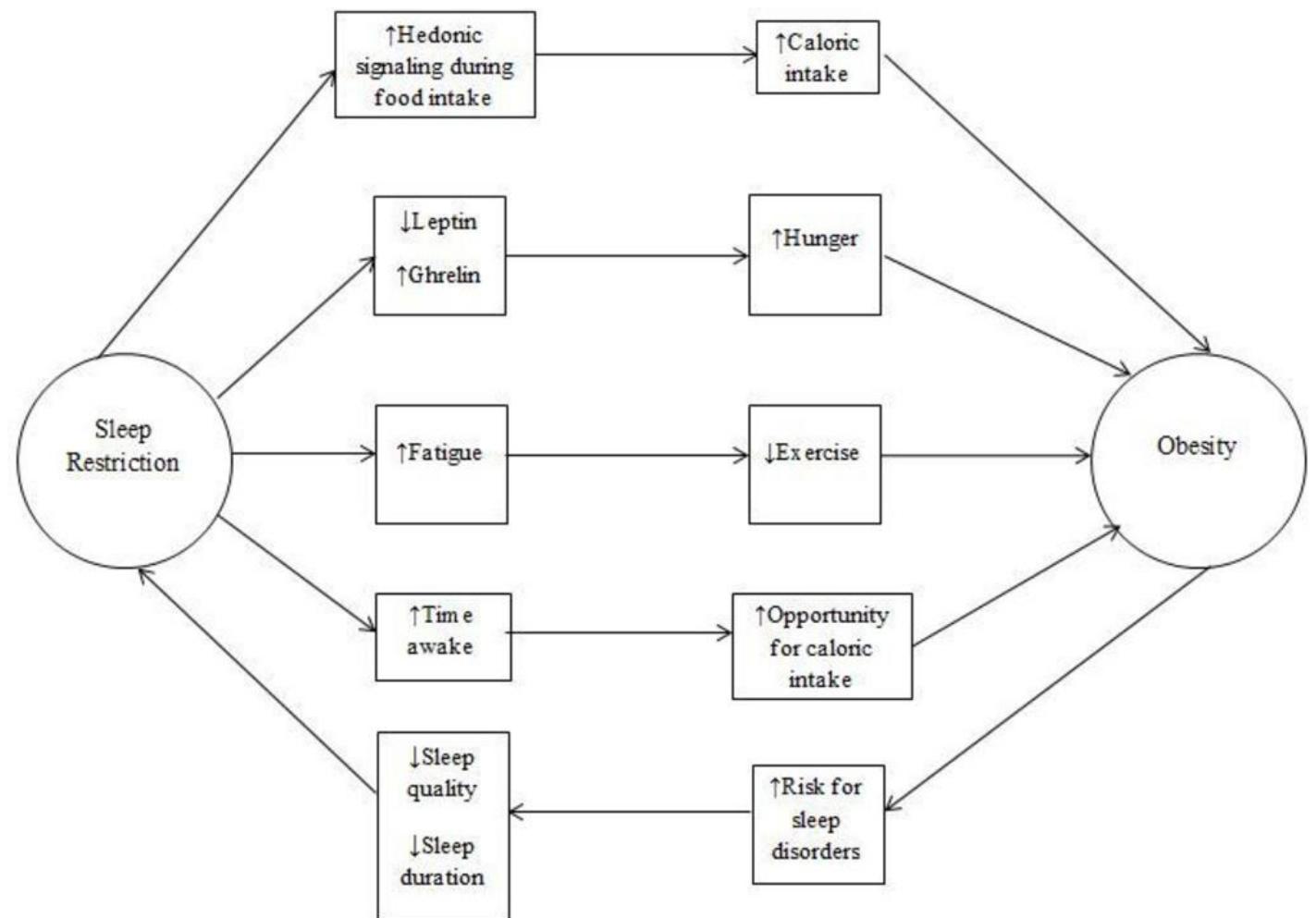

Figure 1 Summary of proposed mechanisms that stimulate the relationship between sleep deprivation and obesity.

unclear whether the decrease in TSH originated directly from the anterior pituitary gland or from decreased secretion of hypothalamic thyrotropin-releasing hormone. Additionally, prolonged short sleep durations could lead to hypertension through chronically raised blood pressure over the 24-hour period, increased salt retention and structural adaptations of the cardiovascular system. ${ }^{34}$ In a report from the Cleveland Family Study, 614 individuals completed questionnaires about sleep habits, underwent polysomnography and had blood tested for circulating markers of inflammation. The conclusion of these authors was that activation of proinflammatory pathways may represent a mechanism by which sleep habits affect health. ${ }^{35}$

While this has not been systematically studied, an additional consideration might be that sleepiness (from insufficient sleep) may lead to decreased physical activity, therefore making weight maintenance more difficult. This behavioural mechanism warrants further research. In addition, there is some evidence that increasing sleep duration may facilitate weight maintenance by decreasing cravings and appetite. ${ }^{36}$ This suggests a potential enhancement to weight management programme may be improvements in sleep duration.

Due to the vastly intricate link between sleep deprivation and obesity, reviewing all literature pertaining to this topic fell beyond the scope of this short review. Instead, its purpose was to provide a framework for understanding mechanisms mediated by hormonal regulators of appetite. Other limitations include the focus on predominantly male adults. Sleep patterns, daily activities and hormone profiles differ between children, adolescents, men, women and the elderly; therefore, some of the mechanisms outlined in the paper may have more or less influence depending on age and sex. While some of the reviewed material involved discussion of both sexes, future investigations should highlight results found in women. Finally, it is important to note that the majority of studies employed self-reported sleep measures rather than objective metrics such as polysomnography or actigraphy. Further research should monitor sleep duration in the home sleep environment.

\section{CONCLUSIONS}

In summary, there is extensive scientific evidence linking sleep restriction to weight gain and obesity. Although mechanistic relationships are not yet clear, if metabolic changes resulting from sleep restriction lead to an increase in body weight, insulin resistance and increased blood pressure, then interventions designed to increase the amount and improve the quality of sleep could serve as treatments and as primary preventative measures for these metabolic disorders. ${ }^{37}$ Further research is needed with objective measures of sleep duration and repeated assessments of both sleep and body weight. Experimental study designs that manipulate sleep duration are also necessary to better explore the possibility of a causal relationship between sleep deprivation and obesity.

Contributors All authors made significant contributions. CBC conceived the idea for this paper and performed the literature review. EVN, BAD and JLM wrote significant portions of the manuscript. 
Funding The authors have not declared a specific grant for this research from any funding agency in the public, commercial or not-for-profit sectors.

Competing interests None declared.

Patient consent Not required.

Provenance and peer review Not commissioned; externally peer reviewed.

Data sharing statement № additional data are available.

Open access This is an open access article distributed in accordance with the Creative Commons Attribution Non Commercial (CC BY-NC 4.0) license, which permits others to distribute, remix, adapt, build upon this work non-commercially, and license their derivative works on different terms, provided the original work is properly cited, appropriate credit is given, any changes made indicated, and the use is non-commercial. See: http://creativecommons.org/licenses/by-nc/4.0/

\section{REFERENCES}

1. Flegal KM, Kruszon-Moran D, Carroll MD, et al. Trends in obesity among adults in the United States, 2005 to 2014. JAMA 2016;315:2284-91.

2. Liu Y, Wheaton AG, Chapman DP, et al. Prevalence of healthy sleep duration among adults--United States, 2014. MMWR Morb Mortal Wkly Rep 2016;65:137-41.

3. Watson NF, Badr MS, Consensus Conference Panel. Joint consensus statement of the american academy of sleep medicine and sleep research society on the recommended amount of sleep for a healthy adult: methodology and discussion. Sleep 2015;38:1161-83.

4. Luckhaupt SE, Tak S, Calvert GM. The prevalence of short sleep duration by industry and occupation in the National Health Interview Survey. Sleep 2010;33:149-59.

5. Cappuccio FP, Taggart FM, Kandala NB, et al. Meta-analysis of short sleep duration and obesity in children and adults. Sleep 2008;31:619-26.

6. Patel SR, Ayas NT, Malhotra MR, et al. A prospective study of sleep duration and mortality risk in women. Sleep 2004;27:440-4.

7. Theorell-Haglöw J, Berglund L, Berne C, et al. Both habitual short sleepers and long sleepers are at greater risk of obesity: a population-based 10-year follow-up in women. Sleep Med 2014;15:1204-11.

8. Xiao $\mathrm{Q}$, Arem H, Moore SC, et al. A large prospective investigation of sleep duration, weight change, and obesity in the NIH-AARP Diet and Health Study cohort. Am J Epidemiol 2013;178:1600-10.

9. Wu Y, Zhai L, Zhang D. Sleep duration and obesity among adults: a meta-analysis of prospective studies. Sleep Med 2014;15:1456-62.

10. Patel SR, Malhotra A, White DP, et al. Association between reduced sleep and weight gain in women. Am J Epidemiol 2006;164:947-54.

11. Andreeva VA, Torres MJ, Léger $D$, et al. Major change in body weight over 5 years and total sleep time: investigation of effect modification by sex and obesity in a large e-cohort. Int $J$ Behav Med 2017;24:493-500.

12. Nielsen LS, Danielsen KV, Sørensen TI. Short sleep duration as a possible cause of obesity: critical analysis of the epidemiological evidence. Obes Rev 2011;12:78-92.

13. Xi B, He D, Zhang M, et al. Short sleep duration predicts risk of metabolic syndrome: a systematic review and meta-analysis. Sleep Med Rev 2014;18:293-7.

14. Gangwisch JE, Malaspina D, Boden-Albala B, et al. Inadequate sleep as a risk factor for obesity: analyses of the NHANES I. Sleep 2005;28:1289-96.

15. Marshall NS, Glozier N, Grunstein RR. Is sleep duration related to obesity? A critical review of the epidemiological evidence. Sleep Med Rev 2008;12:289-98.
16. Klok MD, Jakobsdottir S, Drent ML. The role of leptin and ghrelin in the regulation of food intake and body weight in humans: a review. Obes Rev 2007;8:21-34.

17. Spiegel K, Leproult R, L'hermite-Balériaux M, et al. Leptin levels are dependent on sleep duration: relationships with sympathovagal balance, carbohydrate regulation, cortisol, and thyrotropin. J Clin Endocrinol Metab 2004;89:5762-71.

18. Spiegel K, Tasali E, Penev P, et al. Brief communication: sleep curtailment in healthy young men is associated with decreased leptin levels, elevated ghrelin levels, and increased hunger and appetite. Ann Intern Med 2004;141:846-50.

19. Spiegel K, Knutson K, Leproult R, et al. Sleep loss: a novel risk factor for insulin resistance and Type 2 diabetes. J Appl Physiol 2005;99:2008-19.

20. Penev PD. Sleep deprivation and energy metabolism: to sleep, perchance to eat? Curr Opin Endocrinol Diabetes Obes 2007;14:374-81.

21. St-Onge MP. The role of sleep duration in the regulation of energy balance: effects on energy intakes and expenditure. J Clin Sleep Med 2013;9:73-80.

22. Ogilvie RP, Patel SR. The epidemiology of sleep and obesity. Sleep Health 2017:3:383-8.

23. Dashti HS, Scheer FA, Jacques PF, et al. Short sleep duration and dietary intake: epidemiologic evidence, mechanisms, and health implications. Adv Nutr 2015;6:648-59.

24. Ayas NT, White DP, Al-Delaimy WK, et al. A prospective study of selfreported sleep duration and incident diabetes in women. Diabetes Care 2003;26:380-4

25. Tasali E, Leproult R, Spiegel K. Reduced sleep duration or quality: relationships with insulin resistance and type 2 diabetes. Prog Cardiovasc Dis 2009;51:381-91.

26. Alvarez GG, Ayas NT. The impact of daily sleep duration on health: a review of the literature. Prog Cardiovasc Nurs 2004;19:56-9.

27. Gangwisch JE, Heymsfield SB, Boden-Albala B, et al. Short sleep duration as a risk factor for hypertension: analyses of the first National Health and Nutrition Examination Survey. Hypertension 2006;47:833-9.

28. Cappuccio FP, Cooper D, D'Elia L, et al. Sleep duration predicts cardiovascular outcomes: a systematic review and meta-analysis of prospective studies. Eur Heart J 2011;32:1484-92.

29. Williams CJ, Hu FB, Patel SR, et al. Sleep duration and snoring in relation to biomarkers of cardiovascular disease risk among women with type 2 diabetes. Diabetes Care 2007;30:1233-40.

30. Killick R, Banks S, Liu PY. Implications of sleep restriction and recovery on metabolic outcomes. J Clin Endocrinol Metab 2012;97:3876-90.

31. Bayon V, Leger D, Gomez-Merino D, et al. Sleep debt and obesity. Ann Med 2014;46:264-72.

32. Cedernaes J, Schiöth HB, Benedict C. Determinants of shortened, disrupted, and mistimed sleep and associated metabolic health consequences in healthy humans. Diabetes 2015;64:1073-80.

33. Kessler L, Nedeltcheva A, Imperial J, et al. Changes in serum TSH and free T4 during human sleep restriction. Sleep 2010;33:1115-8.

34. Hasler G, Buysse DJ, Klaghofer R, et al. The association between short sleep duration and obesity in young adults: a 13-year prospective study. Sleep 2004;27:661-6.

35. Patel SR, Zhu X, Storfer-Isser A, et al. Sleep duration and biomarkers of inflammation. Sleep 2009;32:200-4.

36. Tasali E, Chapotot F, Wroblewski K, et al. The effects of extended bedtimes on sleep duration and food desire in overweight young adults: a home-based intervention. Appetite 2014;80:220-4.

37. Gangwisch JE. Epidemiological evidence for the links between sleep, circadian rhythms and metabolism. Obes Rev 2009;10(Suppl 2):37-45 